\title{
ON DISCOVERY OF THE FERROMAGNETIC SUPERCONDUCTOR UCOGe
}

\author{
Nguyen Thanh Huy $^{(1)}$, Dao Duc Cuong ${ }^{(1)}$, Vu Thanh Thu ${ }^{(2)}$, Bui Tu An ${ }^{(1)}$ \\ (1) PetroVietnam University; (2) VNU-HaNoi \\ (Manuscript Received on October $21^{\text {th }}, 2010$, Manuscript Revised January 21 ${ }^{\text {st }}$, 2011)
}

ABSTRACT: We report the coexistence of ferromagnetic order and superconductivity in UCoGe at ambient pressure. The data obtained from the basic thermal, magnetic and transport properties on the macro and microscopic scale show that UCoGe is a weak ferromagnet with a Curie temperature $T_{C}$ $=3 \mathrm{~K}$, and also, is a superconductor with a resistive transition temperature $T_{s}=0.8 \mathrm{~K}$. Those present evidence that UCoGe is an unconventional superconductor and argue that superconductivity is mediated by critical ferromagnetic spin fluctuations.

Keywords: Ferromagnetic superconductor, Ferromagnetic quantum critical point, Critical spin fluctuations.

\section{INTRODUCTION}

In ferromagnetic (FM) state below the Curie temperature, $T_{\mathrm{C}}$, the electron spins align to produce a net magnetization. For a long time it was thought that superconductivity (SC) is incompatible with ferromagnetism. This is rooted in the microscopic theory of superconductivity published in 1957 by Bardeen, Cooper, and Schrieffer (BCS) [1]. Within the standard BCS scenario, a superconducting condensate is formed under the influence of an attractive force due to lattice vibrations which binds electrons with antiparallel spins in singlet Cooper pairs $|\uparrow \downarrow\rangle$ with orbital momentum $L=0$ and projection of the spin momentum $S_{\mathrm{z}}=0$. When magnetic impurity atoms are placed in a conventional superconductor, the local field surrounding the impurity atom suppresses singlet Cooper pair formation, which causes a rapid depression of the $\mathrm{SC}$ transition temperature, $T_{\mathrm{s}}$.
In the year 2000 , the discovery of the first superconducting ferromagnet $\mathrm{UGe}_{2}$ came as a big surprise [2]. In $\mathrm{UGe}_{2}$, superconductivity is realized well below the Curie temperature, without expelling the ferromagnetic order. Since then, three other superconducting ferromagnets have been discovered: UIr [3], URhGe [4], and UCoGe [5]. These materials have in common that ferromagnetic order is due to the uranium $5 f$ magnetic moments and has a strong itinerant character. Moreover, superconductivity occurs close to a magnetic instability. The coexistence of SC and FM in these materials can be understood in terms of spin fluctuation models [6]: in the vicinity of a FM quantum critical point, critical magnetic fluctuations can mediate superconductivity by pairing the electrons in spin-triplet Cooper pairs, that is, the equal spin pairing (ESP) $|\uparrow \uparrow\rangle$ with $L=1, S_{\mathrm{z}}=1,|\downarrow \downarrow\rangle$ with $L=1, S_{\mathrm{z}}=-1$, and the state $(|\uparrow \downarrow\rangle+|\downarrow \uparrow\rangle) / 1.41$ with $L=1, S_{\mathrm{z}}=0$. 
In recent years ample evidence has been presented that such an unusual pairing mechanism is at work in superconducting ferromagnets $[2,7]$.

With the discovery of superconducting ferromagnets a new research theme in the field of magnetism and SC has been disclosed. Research into ferromagnetic superconductors will help to unravel how magnetic fluctuations can stimulate superconductivity, which is a central theme running through materials families as diverse as the heavy-fermion superconductors $[2,4,8]$, high- $T_{\mathrm{s}}$ cuprates [9] and the recently discovered FeAs-based superconductors [10]. This novel insight might turn out to be crucial in the design of new superconducting materials.

UCoGe belongs to the family of intermetallic UTX compounds, with $T$ is a transition metal and $X$ is $\mathrm{Si}$ or $\mathrm{Ge}$, and crystallizes in the orthorhombic TiNiSi structure (space group $P_{\text {nma }}$ ) [11]. From magnetization, resistivity and specific-heat measurements, UCoGe is considered as a paramagnetic ground state down to temperature down 1.2 K $[12,13]$. However, in a search for a FM quantum critical point induced in the ferromagnetic superconductor URhGe $\left(T_{\mathrm{s}}=\right.$ $0.25 \mathrm{~K}, T_{\mathrm{C}}=9.5 \mathrm{~K}$ ) by doping Rh with Co [14], it was discovered that UCoGe is actually a weak itinerant ferromagnet below $T_{\mathrm{C}}=3 \mathrm{~K}$ and, moreover, a superconductor below $T_{\mathrm{s}}=$ $0.8 \mathrm{~K}$, firstly reported in 2007 [5].

In this paper we review the basic thermal, magnetic and transport properties and muon spin relaxation of polycrystalline $\mathrm{UCoGe}$ samples. Magnetization measurements show $\mathrm{UCoGe}$ is a weak itinerant ferromagnet with a Curie temperature $T_{\mathrm{C}}=3 \mathrm{~K}$ and a small ordered moment $m_{0}=0.03 \mu_{\mathrm{B}}$, while $\mathrm{SC}$ is observed with a resistive transition temperature $T_{\mathrm{s}}=0.8$ K. Muon spin relaxation measurements provide unambiguous proof that ferromagnetism is a bulk property, which coexists with superconductivity on the microscopic scale. Since SC occurs right on the borderline of FM, UCoGe may present a typical example of triplet SC stimulated by critical fluctuations associated with a FM quantum critical point (QCP).

\section{EXPERIMENT}

Most experimental methods have been carried out at the Van der Waals - Zeeman Institute (WZI) of the University of Amsterdam. Polycrystalline UCoGe samples were prepared with nominal compositions $\mathrm{U}_{1.02} \mathrm{CoGe}$ by arc melting the constituents (natural U 99.9\%, Co 99.9\%, and Ge 99.999\%) under a high-purity argon atmosphere in a water-cooled copper crucible. The as-cast samples were annealed for 10 days at $875^{\circ} \mathrm{C}$. Samples with typical dimensions of $1 \times 1 \times 6$ $\mathrm{mm}^{3}$ for the different experiments were cut by AGIEPLUS spark erosion, after which the defected surface was removed by polishing. Powder x-ray diffraction patterns at $T=300 \mathrm{~K}$, which were verified by a Philips APD-1700 diffractometer using $\mathrm{Cu}-\mathrm{K}_{\alpha}$ radiation, confirmed the TiNiSi structure. The lattice constants extracted by means of a Rietveld

\section{Trang 22}


refinement using X'pert HighScore Plus to be $a$ $=6.845 \AA, b=4.206 \AA$ and $c=7.222 \AA$, in agreement with literature [11]. The phase homogeneity of the annealed samples was investigated by electron microprobe analysis on JEOL JXA-8621 equipment. The matrix has the $1: 1: 1$ composition and all samples contained a small amount (2\%) of impurity phases.

The dc-magnetization was measured for temperatures $T=2 \mathrm{~K}$ and magnetic fields $B=5$ $\mathrm{T}$ in a Quantum Design SQUID magnetometer MPMS-XL [15]. Four-point low-frequency resistivity and ac-susceptibility data were obtained using a Linear Research AC Bridge Resistance model LR700 operating at a frequency of $16 \mathrm{HZ}$ and low excitation currents $10-100 \mu \mathrm{A}$ in the range $T=0.02-8 \mathrm{~K}$. Thermal expansion data were collected using a capacitance dilatometer for $T=0.23-8 \mathrm{~K}$. Here experiments were carried out in an Oxford Instruments HelioxVL ${ }^{3} \mathrm{He}$ system $\left(T_{\text {base }}=250 \mathrm{mK}\right)$ and an Oxford Instruments Kelvinox MX100 dilution refrigerator $\left(T_{\text {base }}=\right.$ $20 \mathrm{mK})$.

The specific heat was measured in a homebuilt set-up using a semi-adiabatic method employing a mechanical heat switch in a ${ }^{3} \mathrm{He}$ cryostat equipped with a $17 \mathrm{~T}$ superconducting magnet in the temperature range $T=0.5-8 \mathrm{~K}$. Zero-field (ZF) muon spin relaxation $(\mu \mathrm{SR})$ experiments were carried out using the $\mu^{+}$SRdedicated beam line on the PSI- $600 \mathrm{MeV}$ proton accelerator at the Swiss Muon Source of the Paul Scherrer Institute (PSI) in Villigen,
Switzerland in the temperature range $T=0.02$ $-8 \mathrm{~K}$.

\section{RESULT}

\subsection{Weak Itinerant Ferromagnetic} Order

Magnetization data taken on polycrystalline samples provide solid evidence that $\mathrm{UCoGe}$ is a weak itinerant ferromagnet, see Fig. 1. The Curie temperature $T_{\mathrm{C}}=3 \mathrm{~K}$ is deduced from the temperature derivative of the magnetization $\mathrm{d} M(T) / \mathrm{d} T$. A hysteresis loop that has an $S$-shape and exhibit visible remnant moments and coercive fields of $0.3 \mathrm{mT}$ measured at $2 \mathrm{~K}$ further corroborates FM order, see the inset of Fig. 1. The very small size of the ordered moment of $0.03 \mu_{\mathrm{B}}$ is obtained from a smooth extrapolation of the data to $T \rightarrow 0 \mathrm{~K}$. Consequently, the ratio, $p_{\text {eff }} / M_{\mathrm{s}}$, of the CurieWeiss effective moment $p_{\text {eff }}=1.7 \mu_{\mathrm{B}}$ over the saturation moment $M_{\mathrm{s}}$ is small, which classifies UCoGe as a weak itinerant ferromagnet [16].

The temperature dependence of the electrical resistivity of $\mathrm{UCoGe}$ samples is shown in Fig. 2. A broad hump around $3 \mathrm{~K}$ associated with the ferromagnetic transition is observed. In the FM phase $\left(T_{\mathrm{s}}<T<T_{\mathrm{C}}\right)$, the resistivity obeys the relation $\rho \sim T^{2}$. In the temperature range above $T_{\mathrm{C}}\left(T_{\mathrm{C}}<T<3 T_{\mathrm{C}}\right)$ the resistivity is well described by a function $\rho \sim T$ $5 / 3$. The temperature dependence of the resistivity of UCoGe $\left(\sim T^{2}\right.$ and $T^{5 / 3}$ for $T$ below and above $T_{\mathrm{C}}$, respectively) is characteristic for a weak itinerant electron ferromagnet. The $T^{2}$ term below $T_{\mathrm{C}}$ is due to scattering at magnons, while for $T>T_{\mathrm{C}}$ the $T^{5 / 3}$ 
term signals scattering at critical FM spin fluctuations [17]. The resistivity data provide further evidence that UCoGe is near the critical boundary for magnetic long-range order.

The thermodynamic signature of the ferromagnetic transition in the specific heat measured on a polycrystalline sample is shown in Fig. 3. Here $T_{\mathrm{C}}=3 \mathrm{~K}$ is identified by the inflection point in $c / T$ at the high $T$ side of the peak. The linear term in the electronic specific heat $\gamma$ amounts to $0.057 \mathrm{~J} / \mathrm{molK}^{2}$, which indicates $\mathrm{UCoGe}$ is a correlated metal, but the electron interactions are relatively weak. The magnetic entropy $S_{\text {mag }}$ involved in the magnetic transition, obtained by integrating $c_{\text {mag }} / T$ versus $T$, is $0.3 \%$ of $R \ln 2$ (i.e. the value for a local moment $S=1 / 2$ system). Such a small value is expected for a weak itinerant ferromagnet [18].

In order to investigate the weak itinerant ferromagnetism of UCoGe on a microscopic scale, the muon spin relaxation experiments have carried out [19]. The temperature variation $v(T)$ is shown in Fig. 4 and tracks the macroscopic magnetization $M(T)$. For $T \leq T_{\mathrm{C}}$, the data are well described by the relation $v(T)$ $=v_{0}\left(1-\left(T / T^{*}\right)^{\alpha}\right)^{\beta}$ with values of the spontaneous frequency $v_{0}=1.98 \mathrm{MHz}$ for $T \rightarrow$ 0 , the critical temperature $T^{*}=3.02 \mathrm{~K} \approx T_{\mathrm{C}}, \alpha$ $=2.3$, and the critical value $\beta=0.4$ which is close the theoretical value predicted for $3 \mathrm{D}$ Ising-like magnet. The frequency $v \approx v_{0}=2$ $\mathrm{MHz}$ measured at low temperatures, corresponds to an internal field $B_{\mathrm{i}} \sim 0.0148 \mathrm{~T}$ at the muon localization site. These data provide unambiguous proof for magnetic order being present in the whole sample volume. Moreover, magnetic order persists in the superconducting state. Interestingly, in the superconducting state the precession frequency shows a small decrease of about $2 \%$, indicating magnetism and superconductivity interact.

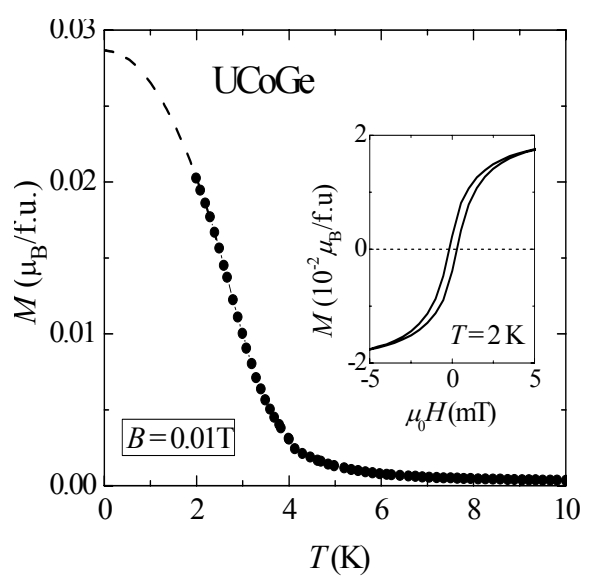

Figure 1. Magnetization of $\mathrm{UCoGe}$ as a function of temperature in a field B of $0.01 \mathrm{~T}$ as indicated. The dashed line is a smooth extrapolation of the data to 0 K. Inset: Hysteresis loop measured at $2 \mathrm{~K}$ in the FM state.

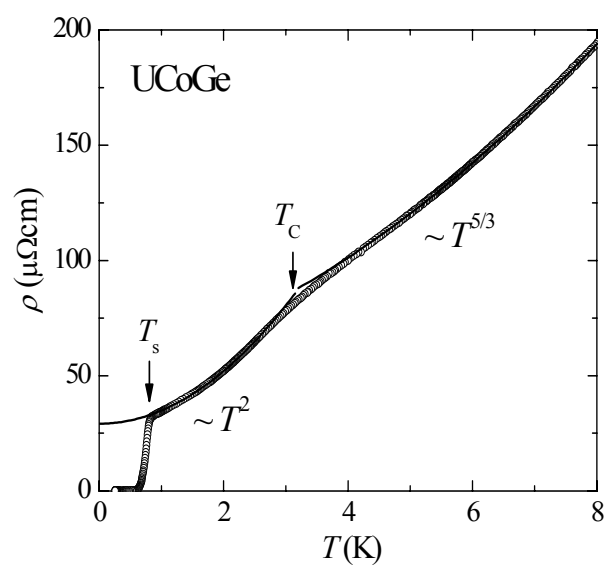

Figure 2. Temperature dependence of the electrical resistivity measured on polycrystalline $\mathrm{UCoGe}$ sample. Arrows indicate the Curie temperature $T_{\mathrm{C}}$

Trang 24 
and superconducting transition temperature $T_{\mathrm{s}}$. The solid lines represent fits of the data to $\rho \sim T^{2}$ and $\sim T$

${ }^{5 / 3}$ in the temperature ranges below and above $T_{\mathrm{C}}$, respectively.

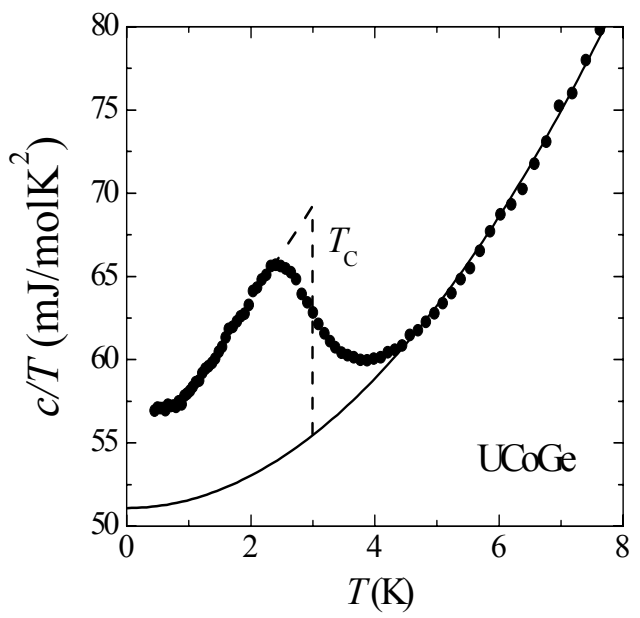

Figure 3. Temperature dependence of the specific heat of UCoGe divided by temperature $\mathrm{c} / \mathrm{T}$ in zero field.

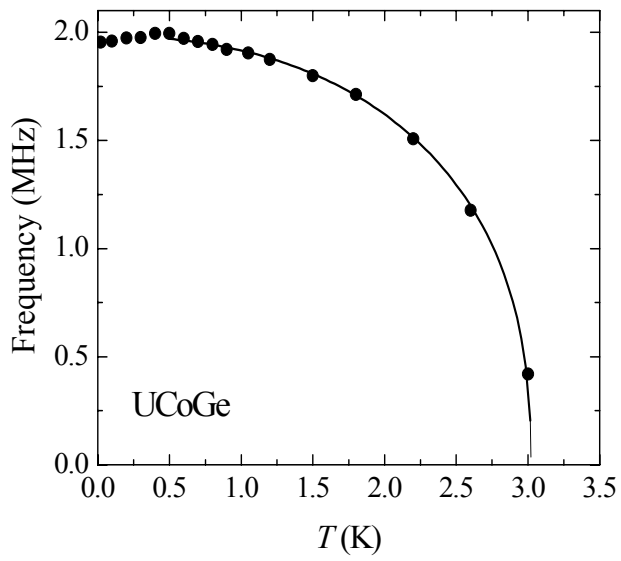

Figure 4. Temperature dependence of the muon precession frequency $v(T)$ of the polycrystalline sample UCoGe in zero magnetic field.

\subsection{Unconventional Superconductivity}

Ac-susceptibility measurements are carried out at a low frequency of $16 \mathrm{~Hz}$ and in a small driving field of $\sim 10^{-5}$ T. Fig. 5 shows the real part of the ac-susceptibility, $\chi_{\text {ac }}^{\prime}$, of polycrystalline $\mathrm{UCoGe}$ as a function of temperature. The weak peak observed at $3 \mathrm{~K}$ reveals the ferromagnetic transition. Below 1 $\mathrm{K}, \chi_{\text {ac }}^{\prime}$ rapidly decreases to a large diamagnetic value, which reflects the superconducting transition. The onset transition temperatures $T_{\text {s,onset }}$ is determined at $0.61 \mathrm{~K}$. Tha result is good agreement with the resistivity data, however, the ac-susceptibility $\chi_{\text {ac }}^{\prime}$ starts to drop when the resistive transition is complete. At the lowest temperature $\chi_{\text {ac }}^{\prime}$ reaches a value of $60 \%$ of the ideal screening value $\chi_{\mathrm{s}}=-1 /(1-N)$ (here $N \approx 0.08$ is the demagnetizing factor of our samples). This indicates UCoGe is a type II SC which is always in the mixed phase. Because of the intrinsic FM moments the local field is nonzero and the magnitude of $\chi_{\text {ac }}^{\prime}$ is reduced.

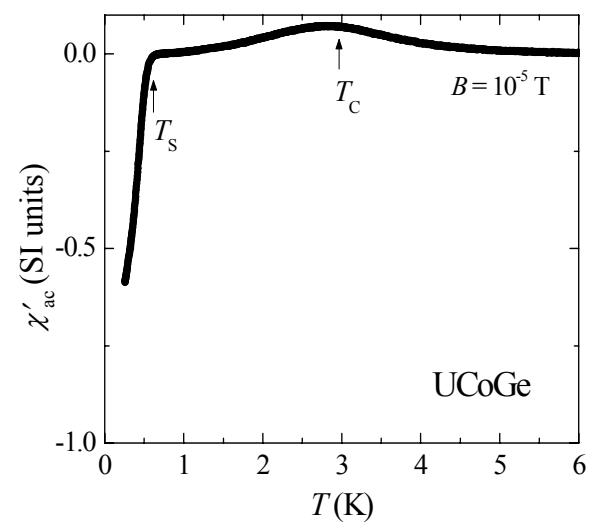

Figure 5. Temperature dependence of the real part of the ac-susceptibility $\chi_{\text {ac }}^{\prime}$ in polycrystalline UCoGe. Arrows indicate $\mathrm{T}_{\mathrm{C}}$ and $\mathrm{T}_{\mathrm{s}}$. 
Specific-heat and thermal-expansion measurements provide solid evidence for bulk superconductivity. Specific-heat data taken on a polycrystalline sample show a broad superconducting transition with an onset temperature of $0.66 \mathrm{~K}$ [5], which is almost equal to the temperature at which the resistance becomes zero. A rough estimate for the step size of the idealized transition in the specific heat, using an equal entropy construction (with a bulk $\left.T_{\mathrm{s}} \approx 0.45 \mathrm{~K}\right)$, yields $\Delta\left(c / T_{\mathrm{s}}\right) / \gamma \approx 1.0$, which is considerably smaller than the BCS value $1.43[1]$.

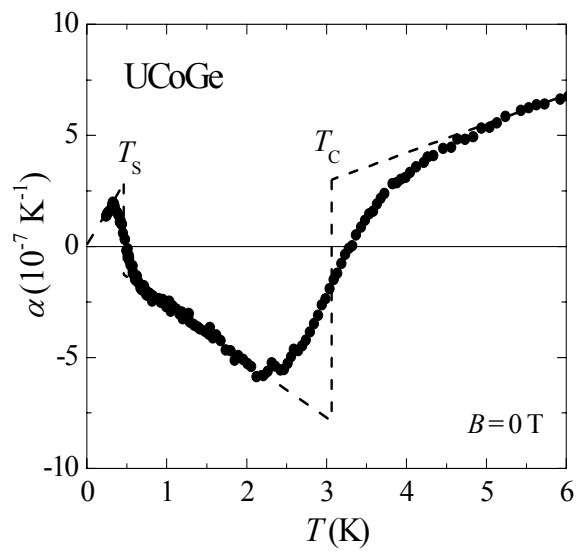

Figure 6. Temperature variation of the coefficient of linear thermal expansion of UCoGe polycrystal.

The linear coefficient of thermal expansion, $\alpha=L^{-1} \mathrm{~d} L / \mathrm{d} T$, measured on a polycrystalline UCoGe is shown in Fig. 6. Upon entering the superconducting state, $\alpha(T)$ shows a steady increase. Assuming an ideal sharp transition at a superconducting temperature $T_{\mathrm{s}}=0.45 \mathrm{~K}$, the estimated stepsize $\Delta \alpha$ is $3.8 \times 10^{-7} \mathrm{~K}^{-1}$ [5], which reflects bulk superconductivity. Moreover, the thermal expansion data reveal that magnetism and superconductivity coexist. The relative length change in the superconducting state $\Delta L / L=$ $-0.1 \times 10^{-6}$ is small compared to the length change $\Delta L / L=1.9 \times 10^{-6}$ due to magnetic ordering. Thus magnetism is not expelled below $T_{\mathrm{s}}$ and coexists with superconductivity. The same conclusion was reached by ${ }^{59} \mathrm{Co}-$ NQR measurements on poly and singlecrystalline samples: below $T_{\mathrm{C}} \approx 2.5 \mathrm{~K}$ ferromagnetism and superconductivity are found to coexist on the microscopic scale [20]. From the temperature variation of the NQR spectrum, the authors conclude that the ferromagnetic phase transition is weakly first order.

Following the discovery that polycrystalline compound $\mathrm{UCoGe}$ is a new ambient pressure ferromagnetic superconductor, the study on UCoGe singlecrystal have carried out [21]. Magnetization measurements show that UCoGe is a uniaxial FM, with $T_{\mathrm{C}}=2.8 \mathrm{~K}$ and an ordered moment $m_{0}=0.07 \mu_{\mathrm{B}}$ pointing along the orthorhombic $c$ axis, and superconductivity occurs below $T_{\mathrm{s}}=$ $0.65 \mathrm{~K}$. Measurements of the upper critical field $B_{\mathrm{c} 2}$ support triplet superconductivity and point to an axial superconducting gap function with nodes along the $c$-axis, that is, the direction of the ordered moment $m_{0}$. The $B_{\mathrm{c} 2}$ curves show an unusual upward curvature $(B \|$ $b$ ) or kink ( $B \| a$ ), which is possibly due to a competition between the equal-spin pairing states $|\uparrow \uparrow\rangle$ and $|\downarrow \downarrow\rangle$, expected for a twoband ferromagnetic superconductor [21]. Under

Trang 26 
hydrostatic pressure, ferromagnetism is depressed vanishes near critical pressure $p_{\mathrm{c}}=$ 1.4 GPa, while superconductivity is enhanced and survives in the paramagnetic phase at least up to $2.2 \mathrm{GP}$ [22]. However, for $p>1.0 \mathrm{GPa}$, ferromagnetic order is no longer observed. The $p-T$ phase diagram reveals superconductivity is closely connected to a ferromagnetic QCP hidden under the superconducting "dome".

\section{CONCLUSION}

In this paper we have reviewed the thermal, magnetic, and transport properties of the polycrystalline superconducting ferromagnet UCoGe. The data provide solid evidence for bulk superconductivity below 0.8 $\mathrm{K}$, which coexists with bulk weak itinerant ferromagnetism with a Curie temperature of 3 K. Since SC occurs right on the borderline of FM order at ambient pressure, UCoGe offers a unique testing ground to investigate the longstanding issue of SC stimulated by critical spinfluctuations associated with a magnetic quantum critical point.

\section{HợP CHẤT SIÊU DÃ̃N SĂT TÙ̉ UCoGe}

\section{Nguyễn Thành Huy ${ }^{(1)}$, Đào Đức Cường ${ }^{(1)}$, Vũ Thanh Thu ${ }^{(2)}$, Bùi Tử An ${ }^{(1)}$}

(1) Trường Đại học Dầu khí Việt Nam; (2) ĐHQG Hà Nội

TÓM TÁT: Chúng tôi trình bày sụ xuất hiện đồng thời của trật tụ sắt tù và trạng thái siêu dân trong hơp kim UCoGe tại áp suất khi quyển. Số liệu thực nghiệm thu được tù các phép đo co bản đặc trung của các tính chất tù̀, nhiệt, điện cho thấy UCoGe là chất sắt tù̀ yếu với nhiệt độ Curie của chuyển pha sắt tù - thuận tù $T_{C}=3 \mathrm{~K}$, và là vật liệu sắt tù với nhiệt độ chuyển pha siêu dẫn $T_{s}=0.8 \mathrm{~K}$. Các kết quả này chứng tỏ UCoGe là một vật liệu siêu dẫn dị thường trong đó trạng thái siêu dẫn có thể được gây nên bởi thăng giáng tới hạn của spin gần chuyển pha luợng tử sắt tù - thuận tù tại nhiệt độ $0 \mathrm{~K}$.

Tù khoá: Vật liệu siêu dẫn sắt tù, Điểm tới hạn luợng tử, Thăng giáng spin tói hạn.

\section{REFERENCE}

[1]. J. Bardeen, L.N. Cooper and J.R. Schrieffer, Phys. Rev. 108 (1957) 1175.

[2]. S.S. Saxena, K. Ahilan, P.Agarwal, F.M. Grosche, R.K. Haselwimmer, M. Steiner, E. Pugh, I.R. Walker, S.R. Julian, P. Monthoux, G.G. Lonzarich, A.D. Huxley, I. Sheikin, D. Braithwaite and J.
Flouquet, Nature (London) 406 (2000) 587.

[3]. T. Akazawa, H. Hidaka, T. Fujiwara, T.C. Kobayashi, E. Yamamoto, Y. Haga, R. Settai and Y. Ōnuki, J. Phys. Condens. Matter 16 (2004) L29.

[4]. D. Aoki, A.D. Huxley, E. Ressouche, D. Braithwaite, J. Flouquet, 
J.P. Brison, E. Lhotel and C. Paulsen, Nature (London) 413 (2001) 613.

[5]. N.T. Huy, A. Gasparini, D.E. de Nijs, Y. Huang, J.C.P. Klaasse, T. Gortenmulder, A. de Visser, A. Hamann, T. Görlach and H. v. Löhneysen, Phys. Rev. Lett. 99 (2007) 067006.

[6]. D. Fay and J. Appel, Phys. Rev. B 22 (1980) 3173.

[7]. F. Lévy, I. Sheikin and A.D. Huxley, Nature Physics 3 (2007) 460.

[8]. N.D. Mathur, F.M. Grosche, S.R. Julian, I.R. Walker, D.M. Freye, R.K.W. Haselwimmer and G.G. Lonzarich, Nature (London) 394 (1998) 39.

[9]. J.G. Bednorz and K.A. Müller, $Z$. Phys. B 64 (1986) 189.

[10]. Y. Kamihara, T. Watanabe, M. Hirano, H. Hosono, J. Am. Chem. Soc. 130 (2008) 3296.

[11]. F. Canepa, P. Manfrinetti, M. Pani and A. Palenzona, J. Alloys Comp. 234 (1996) 225.

[12]. R. Tróc and V.H. Tran, J. Magn. Magn. Mat. 73 (1988) 389.

[13]. K.H.J. Buschow, E. Brück, R.G. van Wierst, F.R. de Boer, L. Havela, V. Sechovský, P. Nozar, E. Sugiura, M. Ono, M. Date and A. Yamagishi, J. Appl. Phys. 67 (1990) 5215.
[14]. S. Sakarya, N.T. Huy, N.H. van Dijk, A. de Visser, J.C.P. Klaasse, M. Wagemaker, T.J. Gortenmulder, M. Uhlarz and H. von Löhneysen, J. Alloys Comp. 457 (2008) 51.

[15]. http://www.qdusa.com/products/mp ms.html

[16]. P.R. Rhodes and E.P. Wohlfarth, Proc. R. Soc. London 273 (1963) 247.

[17]. T. Moriya, Spin Fluctuations in Itinerant Electron Magnetism (Springer, Berlin, 1985).

[18]. E.P. Wohlfarth, Physica $B+C 91$ (1977) 305.

[19]. A. de Visser, N.T. Huy, A. Gasparini, D.E. de Nijs, D. Andreica, C. Baines and A. Amato, Phys. Rev. Lett. 102 (2009) 167003 .

[20]. T. Ohta, T. Hattori, K. Ishida, Y. Nakai, E. Osaki, K. Deguchi, N.K. Sato, I. Satoh, J. Phys. Soc. Jpn. 79 (2010) 023707.

[21]. N.T. Huy, D.E. de Nijs, Y.K. Huang and A. de Visser, Phys. Rev. Lett. 100 (2008) 077002.

[22]. E. Slooten, T. Naka, A. Gasparini, Y.K. Huang, A. de Visser, Phys. Rev. Lett. 103 (2009) 097003.

\section{Trang 28}

